\title{
CORRELATION OF VISUAL AND RADIOGRAPHIC CARIES DIAGNOSIS USING THE ICDAS IN PERMANENT MOLARS OF A GROUP OF EGYPTIAN CHILDREN
}

\author{
Nora Saif*
}

\begin{abstract}
ICDAS (International Caries Detection and Assessment System) is an evidence-based detection and assessment system classifying stages of the caries process on the basis of histological extent and activity. The ICDAS measures the visual surface changes which corresponds to the potential histological depth of carious lesions. In addition, it proposes the ICDAS radiographic scoring system, which categorizes lesions according to the depth of caries demineralization into enamel and dentin represented by a radiolucency on the radiographs. In this study, the aim was to assess the relationship between clinical and radiographic caries diagnoses among 12 to 14-year-old Egyptians using the ICDAS scoring system which differentiates between cavitated and non-cavitated caries lesions.
\end{abstract}

KEYWORDS: ICDAS, caries demineralization, bite wing x-ray, non-parametric statistics.

\section{INTRODUCTION}

Applying a standardized caries detection system based on best evidence ensures better quality information which aids the practitioner in making an informed decision about the best clinical management of dental caries at both the individual and public health levels.

ICDAS (International Caries Detection and Assessment System) is a simple, logical, evidencebased detection and assessment system classifying stages of the caries process on the basis of histological extent and activity. Since its inception in 2001, it has gained wide acceptance and application in the four domains of clinical practice, education, research and public health, providing all stakeholders with a common language for staging caries. ${ }^{[1]} \mathrm{It}$ moves the profession away from the simplistic diagnosis of "No obvious decay" or "Obvious decay" to a more detailed level of diagnosis to improve patient care and more precisely monitor caries development in research. ${ }^{[2]}$

\footnotetext{
* Lecturer, Oral \& Maxillofacial Radiology Dept, Faculty of Oral \& Dental Medicine, Cairo University.
} 
The ICDAS measures the visual surface changes which corresponds to the potential histological depth of carious lesions by relying on visually detected surface characteristics. In addition, for a more comprehensive diagnosis, it proposes the ICDAS radiographic scoring system, which categorizes lesions according to the depth of caries demineralization into enamel and dentin represented by a radiolucency on the radiographs..$^{[3]}$

Clinical visual detection and assessment of lesions on clean dry teeth represents the corner stone for clinical assessment of the carious lesion while radiography remains the most widely used caries detection aid. ${ }^{[1]}$

The bitewing technique is designed to detect proximal caries lesions that cannot be detected by visual inspection. The current literature shows that the use of radiographs is more sensitive than clinical inspection for detecting proximal lesions as well as occlusal lesions in dentin, for estimating depth of the lesion, and for monitoring lesion behavior. ${ }^{[4,5]}$

Radiographically, the ICDAS classifies posterior tooth surfaces in various degrees according to progression, enabling good reproducibility and accuracy of this classification system, which leads to more comprehensive information to plan, manage, and properly diagnose tooth decay. ${ }^{[3,6-8]}$

The aim of this study was to conduct an in vivo investigation of the correlation between the visual and radiographic scoring systems proposed by the ICDAS for the diagnosis of proximal and occlusal caries in the permanent molars of a group of Egyptian children.

\section{MATERIALS AND METHODS}

The study sample comprised 480 occlusal and proximal surfaces from 28 patients referred for treatment at a private pediatric dentistry center. To be included in the study, patients had to be between
12 and 14 yrs, have at least one permanent molar that met the inclusion criteria which are: to be fully erupted and present no restoration on the occlusal surface and proximal surfaces.

All 28 patients received a clinical examination followed by a 2 bitewing radiographs, that were taken and viewed separately and blind. Proximal and occlusal surfaces of molars and premolars were examined on the radiographs.

\section{Visual Inspection}

Visual caries detection of molars was performed using ICDAS criteria ${ }^{[8]}$. ICDAS scores 1 and 2 were recorded as "initial lesions" and scores 3-6 as "advanced lesions" (Table 1). Calculus and plaque were removed if deemed necessary by the dentist. A standard dental mirror and a ball-ended dental probe were used. Teeth were dried for the clinical examination. The data was entered by a dentist standardized in this index, with a 0.80 Kappa index. ${ }^{[7]}$

\section{Radiographic Examination}

Radiographic bitewing caries detection was performed for both occlusal and proximal surfaces. Kodak Intra-oral E-speed films were exposed for $0.12 \mathrm{~s}$ at $70 \mathrm{kVp}$ and $7 \mathrm{~mA}$, and then manually developed as per the manufacturer's instructions. All the dental films were manually processed following the manufacturer's instructions and the same procedure (Develop for $3 \mathrm{~min}$ at $24.5^{\circ} \mathrm{C}$. Rinse under fresh running water at $25-29^{\circ} \mathrm{C}$ for $30 \mathrm{~s}$ with continuous agitation. Fix for $2-4 \mathrm{~min}$ at $24.5^{\circ} \mathrm{C}$. Finally, wash for $10 \mathrm{~min}$ under clean running water at $25-29^{\circ} \mathrm{C}$. Carestream Dental GBX developer and fixer were used)

The radiographic analysis was performed by two dentists previously standardized as inter- and intraexaminer, in the radiographic system proposed by ICDAS, with a 0.80 Kappa index. ${ }^{[7]}$ The detected 
TABLE (1) ICDAS Scores and Radiographic classification of lesion severity

\begin{tabular}{|c|l|l|}
\hline Score & \multicolumn{1}{|c|}{ Visual Criteria } & \multicolumn{1}{c|}{ Radiographic Criteria } \\
\hline 0 & Sound & No radiolucency \\
\hline 1 & First Visual Change in enamel & Radiolucency in outer $1 / 2$ of the enamel \\
\hline 2 & Distinct Visual Change in enamel & Radiolucency in inner $1 / 2$ of the enamel \pm EDJ \\
\hline 3 & Localized enamel breakdown & Radiolucency limited to the outer $1 / 3$ of dentine \\
\hline 4 & Underlying dentine shadow & Radiolucency reaching the middle $1 / 3$ of dentine \\
\hline 5 & Distinct cavity with visible dentine & Radiolucency reaching the inner $1 / 3$ of dentine \\
\hline 6 & Extensive cavity with visible dentine & Radiolucency into the pulp \\
\hline
\end{tabular}

lesions on radiographs were given a score as per the radiographic ICDAS Caries Scoring System, with scores 0-2 recorded as "initial lesions" and scores 3-6 as "advanced lesions" (Table 1).

\section{Statistical Analysis}

Data was statistically described in terms of frequencies (number of cases) and percentages. Agreement between visual and radiographic variables was tested using kappa statistic. Two sided $\mathrm{p}$ values less than 0.05 was considered statistically significant.

All statistical calculations were done using computer program IBM SPSS (Statistical Package for the Social Science; IBM Corp, Armonk, NY, USA) release 22 for Microsoft Windows.

\section{RESULTS}

The comparison between clinical and radiographic diagnosis of the detected occlusal initial carious lesion (Table 2), showed clinical and radiographic coincidence of a healthy diagnosis in $93.3 \%$ of cases. However, $6.66 \%$ of clinically detectable lesions in the initial stages were recorded as healthy from a radiographic point of view.

For the advanced occlusal caries, shows clinical and radiographic coincidence of $95.5 \%$ of the examined surfaces that clinically tested negatively and $37.5 \%$ coincidence when a positive clinical diagnosis of occlusal advanced caries was made (Table 2). The kappa test shows a significant agreement between the clinical and radiographic diagnosis (Table 3).

For proximal caries, (Table 2) demonstrates 95\% agreement between clinical and radiographic diagnosis when excluding an initial proximal caries lesion.

As for advanced proximal caries, (Table 2) shows a $95.6 \%$ agreement between the clinical and radiographic diagnosis when a diagnosis of advanced proximal caries is established and 50\% agreement when it is not. Statistically, there is a strong agreement among them as per the results of the kappa test (Table 3) 
TABLE (2) Clinical \& radiographic correlation of detected caries lesions

\begin{tabular}{|c|c|c|c|c|c|}
\hline & \multirow{2}{*}{\multicolumn{2}{|c|}{ Caries Lesion }} & \multicolumn{2}{|c|}{ Radiographic Diagnosis } & \multirow{2}{*}{ Total } \\
\hline & & & +ve & -ve & \\
\hline & & & cclusal ( & & \\
\hline & & Count & 112 & 0 & 112 \\
\hline & re & $\%$ of coincidence & $100 \%$ & 0 & $100 \%$ \\
\hline & & Count & 8 & 0 & 8 \\
\hline & re & $\%$ of coincidence & $100 \%$ & 0 & $100 \%$ \\
\hline & & & Occlusa & 3-6) & \\
\hline & & Count & 84 & 4 & 88 \\
\hline & ve & $\%$ of coincidence & $95.5 \%$ & $4.5 \%$ & $100 \%$ \\
\hline & & Count & 20 & 12 & 32 \\
\hline & 海 & $\%$ of coincidence & $62.5 \%$ & $37.5 \%$ & $100 \%$ \\
\hline 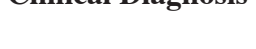 & & & oximal & & \\
\hline & & Count & 113 & 6 & 119 \\
\hline & re & $\%$ of coincidence & $95.0 \%$ & $5.0 \%$ & $100 \%$ \\
\hline & & Count & 1 & 0 & 1 \\
\hline & 政 & $\%$ of coincidence & $100 \%$ & $0 \%$ & $100 \%$ \\
\hline & & & Proxima & 1,2) & \\
\hline & & Count & 109 & 5 & 114 \\
\hline & $=$ & $\%$ of coincidence & $95.6 \%$ & $4.4 \%$ & $100 \%$ \\
\hline & & Count & 3 & 3 & 6 \\
\hline & 政 & $\%$ of coincidence & $50 \%$ & $50 \%$ & $100 \%$ \\
\hline
\end{tabular}

(Table 3) for the symmetric measures and agreement between clinical and radiographic diagnoses, show a significant agreement between the two diagnostic indicators for the advanced lesions in both occlusal and proximal surfaces.

TABLE (3) Agreement between visual and radiographic variables.kappa statistic.

\begin{tabular}{|c|c|c|}
\hline $\begin{array}{c}\text { Measure of Agreement } \\
\text { Kappa }\end{array}$ & Value & p value \\
\hline Occlusal Initial & (a) & ------- \\
\hline Occlusal Advanced & 0.392 & 0.000 \\
\hline Proximal Initial & -0.014 & 0.818 \\
\hline Proximal Advanced & 0.394 & 0.000 \\
\hline
\end{tabular}

\section{Symmetric Measures}

(a) No statistics are computed because radiographic diagnosis for initial occlusal caries (ICDAS 1,2) is a constant.

\section{DISCUSSION}

Detection of caries lesions at an early stage is a necessity for effective prevention of disease. The optimum goal is to classify the lesions according to their biological representation in order to provide the best biologically oriented treatment and thus preserve tooth structure, such that non-cavitated lesions can be controlled with preventive therapies whereas cavitated lesions will require a restoration. 
The International Consensus Workshop on Caries Clinical Trials (ICW-CCT) ${ }^{[9]}$ concluded that:

- Visual diagnosis is the standard of caries diagnosis; the use of additional methods should be explored further.

- Bitewing radiography adds information to the diagnosis.

ICDAS has proven to be an accurate and reproducible method to visually detect and stage caries lesions ${ }^{[10,11]}$. Bite-wing radiography has been recommended for use in adolescents as clinical examinations alone may lead to underestimation of carious lesions in proximal and occlusal surfaces. ${ }^{[12,13]}$

In this study, the aim was to assess the relationship between clinical and radiographic caries diagnoses among 12 to 14-year-old Egyptians using the ICDAS scoring system which differentiates between cavitated and non-cavitated caries lesions.

Previous studies found radiographs to be helpful in the assessment of proximal lesions, although not very early ones, while their use in occlusal detection of early lesions was found to be less optimal than visual inspection and is thus generally restricted to dentine caries and its staging. ${ }^{[4,5]}$

Accordingly, upon comparing the two diagnostic methods, we have separated the occlusal lesions from proximal ones. This separation is based on the prior assumption that, in occlusal surfaces, the contribution of the radiographs seems to be minimal [12]. On the other hand, the literature shows that radiography can add information about the clinical stages of the caries process at proximal surfaces as well as the more advanced stages on occlusal surfaces. ${ }^{[14]}$

In vivo understanding of the relationship between visual clinical examination and bitewing radiographic examination in clinical situations where the surface enamel is non-cavitated, but where dentin is already involved, is of added importance for making a proper diagnosis and treatment decision. ${ }^{[15]}$ Accordingly, the current study also separated the comparison between initial and advanced lesions for both categories, occlusal and proximal caries.

The results showed lack of agreement between the clinical and radiographic diagnosis of initial caries for both occlusal and proximal carious lesions. This can be attributed to the minimal mineral loss in early lesions, which cannot be radiographically perceived. It has been reported that 30 to $40 \%$ of mineral loss is necessary for the radiographic detection of enamel caries. ${ }^{[2]}$. In addition, the overlying of enamel in the occlusal area can mask the mineral loss process.

These findings agree with Wenzel and Fejrskov who stated that radiographs are not useful to detect enamel caries on the occlusal surface, but that they are useful to detect dentine caries on the occlusal surface ${ }^{[16]}$ as well as many other studies that found bitewing radiography to have limitations in the detection of proximal and occlusal enamel caries when compared to meticulous visual inspection and considered it generally more suitable for the detection of dentin caries than for enamel caries. ${ }^{[12,13,17,18]}$

It is worth mentioning, that $100 \%$ of initial occlusal caries and $95 \%$ of initial proximal caries classified as clinically healthy, were also classified as healthy radiographically as per the ICDAS standards. An important observation is that in this study no hidden caries lesions were found, which are those caries characterized as seemingly clinically healthy and that are diagnosed by radiography. This finding is in agreement with the studies of Wolwacz et al. ${ }^{[15]}$, Ekstrand et al. ${ }^{[7]}$ and Machiulskiene et al. ${ }^{[12]}$ Accordingly, visual examination, solely, as per the ICDAS for initial caries detection in this age group is considered reliable enough with no proven added value for further radiographic examination. 
Some studies have shown the value of bitewing radiographs in detecting initial proximal caries, however in the current study we were unable to establish this correlation, due to the low number of cases exhibiting clinical initial proximal caries. The results of our study, is in conjunction with those by Marquezan et al ${ }^{[19]}$ as well as Bertella et al. ${ }^{[20]}$ who suggested that the lack of association between enamel changes associated with initial proximal caries and radiolucency could be attributed to issues related to the sample size and statistical power.

Moving on to the advanced caries comparisons and tables, the results showed an evident significant agreement between clinical and radiographic diagnosis of advanced caries in both occlusal and proximal surfaces, where $37.5 \%$ of the clinically diagnosed advanced occlusal caries recorded the same score radiographically. This percentage increased for advanced proximal caries to reach $50 \%$.

These findings agree with Wolwacz et al. ${ }^{[15]}$ who found a strong correlation between the scores of visual and radiographic diagnostic systems of occlusal caries. Ekstrand et al. ${ }^{[7]}$ suggested that radiographic detection resembles the demineralized area found in the histological examination when the lesion reaches the dentin, and can thus be considered a predictor of softened and demineralized dentin.

Similarly, the study by Wolwacz et al. ${ }^{[15]}$ showed that radiographs are an excellent method to detect softened and infected dentin, mainly in the middle or inner thirds of the dentin although it presents diagnostic failures in the outer third of the dentin and in enamel.

Clinical diagnosis of proximal caries is arguably more problematic due the wide contact areas that render the inter-proximal caries-susceptible area clinically invisible, which makes the radiographic detection and staging of proximal caries of added value in the clinical practice. ${ }^{[21]}$ Poorterman et al. stated that the extra diagnostic yield of bitewings varied between $163 \%$ and $700 \%$ for proximal dentine caries diagnosis and reported a substantial underestimation of the prevalence of proximal caries lesions, and to a lesser extent on occlusal surfaces, by clinical examination alone. ${ }^{[22]}$ Accordingly, radiographic examination for advanced occlusal and proximal lesions has proved to add significantly to clinical findings in terms of defining lesions at different stages of progression. ${ }^{[22-27]}$

Radiography can thus add information about the clinical stages of the caries process at the advanced stages on both proximal and occlusal surfaces.

\section{CONCLUSION}

Visual examination using the ICDAS scoring system proved to be a reliable method for caries detection for initial caries lesions in both occlusal and proximal surfaces, thus providing a simple and standardized way to distinguish between cavitated and non-cavitated caries lesions in clinical practice.

Applying the ICDAS radiographic scoring as a diagnostic aid to the visual scores was found to be beneficial in detecting and staging advanced caries lesions in both occlusal and proximal surfaces.

\section{REFERENCE}

1. Ekstrand, K.R., et al.: The International Caries Detection and Assessment System - ICDAS: A Systematic Review. Caries Res, 2018. 52(5): p. 406-419.

2. Pitts, N.B., et al., Dental caries. Nat Rev Dis Primers, 2017. 3: p. 17030

3. Ismail, A.I., et al.: The International Caries Classification and Management System (ICCMS) An Example of a Caries Management Pathway. BMC Oral Health, 2015. 15 Suppl 1: p. S9.

4. Bader, J.D., D.A. Shugars, and A.J. Bonito: A systematic review of the performance of methods for identifying carious lesions. J Public Health Dent, 2002. 62(4): p. 201-13.

5. Gomez, J., et al.: Non-cavitated carious lesions detection methods: a systematic review. Community Dent Oral Epidemiol, 2013.41(1): p. 54-66. 
6. Ricketts, D.N., et al.: Relating visual and radiographic ranked scoring systems for occlusal caries detection to histological and microbiological evidence. Oper Dent, 2002. 27(3): p. 231-7.

7. Ekstrand, K.R., D.N. Ricketts, and E.A. Kidd: Reproducibility and accuracy of three methods for assessment of demineralization depth of the occlusal surface: an in vitro examination. Caries Res, 1997. 31(3): p. 224-31.

8. NB Pitts, A.I., GVA Douglas: ICCMS ${ }^{\mathrm{TM}}$ Guide for Practitioners and Educators. 2014, www.iccms-web.com.

9. Pitts, N.B. and J.W. Stamm: International Consensus Workshop on Caries Clinical Trials (ICW-CCT)--final consensus statements: agreeing where the evidence leads. J Dent Res, 2004. 83 Spec No C: p. C125-8.

10. Jablonski-Momeni, A., et al.: Reproducibility and accuracy of the ICDAS-II for detection of occlusal caries in vitro. Caries Res, 2008. 42(2): p. 79-87.

11. Ferreira Zandona, A., et al.: Use of ICDAS combined with quantitative light-induced fluorescence as a caries detection method. Caries Res, 2010. 44(3): p. 317-22.

12. Machiulskiene, V., B. Nyvad, and V. Baelum: A comparison of clinical and radiographic caries diagnoses in posterior teeth of 12-year-old Lithuanian children. Caries Res, 1999. 33(5): p. 340-8.

13. Machiulskiene, V., B. Nyvad, and V. Baelum: Comparison of diagnostic yields of clinical and radiographic caries examinations in children of different age. Eur J Paediatr Dent, 2004. 5(3): p. 157-62.

14. Wenzel, A. and L.L. Kirkevang: Students' attitudes to digital radiography and measurement accuracy of two digital systems in connection with root canal treatment. Eur J Dent Educ, 2004. 8(4): p. 167-71.

15. Wolwacz, V.F., et al.: Correlation between visual and radiographic examinations of non-cavitated occlusal caries lesions - an in vivo study. Braz Oral Res, 2004. 18(2): p.145-9.

16. Wenzel, A. and O. Fejerskov: Validity of diagnosis of questionable caries lesions in occlusal surfaces of extract- ed third molars. Caries Res, 1992. 26(3): p. 188-94.

17. Huth, K.C., et al.: In vivo performance of a laser fluorescence device for the approximal detection of caries in permanent molars. J Dent, 2010. 38(12): p. 1019-26.

18. Wenzel, A.: Bitewing and digital bitewing radiography for detection of caries lesions. J Dent Res, 2004. 83 Spec No C: p. C72-5.

19. Marquezan, P.K., et al.: Radiographic pattern of underlying dentin lesions (ICDAS 4) in permanent teeth. Clin Oral Investig, 2019.

20. Bertella, N., et al.: Clinical and radiographic diagnosis of underlying dark shadow from dentin (ICDAS 4) in permanent molars. Caries Res, 2013. 47(5): p. 429-32.

21. Hopcraft, M.S. and M.V. Morgan: Comparison of radiographic and clinical diagnosis of approximal and occlusal dental caries in a young adult population. Community Dent Oral Epidemiol, 2005. 33(3): p. 212-8.

22. Poorterman, J.H., E.H. Vermaire, and J. Hoogstraten: Value of bitewing radiographs for detecting approximal caries in 6-year-old children in the Netherlands. Int J Paediatr Dent, 2010. 20(5): p. 336-40.

23. Poorterman, J.H., I.H. Aartman, and H. Kalsbeek: Underestimation of the prevalence of approximal caries and inadequate restorations in a clinical epidemiological study. Community Dent Oral Epidemiol, 1999. 27(5): p. 331-7.

24. Poorterman, J.H., et al.: Approximal caries increment: a three-year longitudinal radiographic study. Int Dent J, 2003. 53(5): p. 269-74.

25. Poorterman, J.H., et al.: Variations among dentists in the diagnosis of caries and assessment of dental restorations. Ned Tijdschr Tandheelkd, 1997. 104(6): p. 214-8.

26. Poorterman, J.H., et al.: Radiographic dentinal caries and its progression in occlusal surfaces in Dutch 17-year-olds: a 6-year longitudinal study. Caries Res, 2003. 37(1): p.29-33.

27. Poorterman, J.H., et al.: Clinical and radiographic judgement of occlusal caries in adolescents. Eur J Oral Sci, 2000. 108(2): p. 93-8. 